\title{
Inadequate identification of fatty liver disease, obesity, and metabolic syndrome by family physicians
}

This article was published in the following Dove Press journal:

Diabetes, Metabolic Syndrome and Obesity:Targets and Therapy

\author{
Mahmud Mahamid ${ }^{1-3, *}$ \\ Tawfik Khoury,* \\ Hana Amara' \\ Mahmoud Siadi 1,3 \\ Jabaren Mohamed ${ }^{4}$ \\ Amir Mari' \\ Rafea Shalabi ${ }^{5}$ \\ Hisham Sholy ${ }^{6}$ \\ Wiliam Nseir ${ }^{3}$
}

'Gastroenterology and Liver Diseases Unit, EEMS, The Nazareth Hospital, Nazareth, Israel; ${ }^{2}$ Faculty of Medicine in the Galilee, Bar-Ilan University, Safed, Israel; ${ }^{3}$ Division of Internal Medicine, EMMS, The Nazareth Hospital, Nazareth, Israel; ${ }^{4}$ Cardiology Department, HaEmek Medical Center, Afula, Israel; ${ }^{5}$ Department of Internal Medicine, The Baruch Padeh Medical Center, Poriya, Israel; 'Liver Unit, Rambam Health Campus, Haifa, Israel

*These authors contributed equally to this work

Correspondence: Hana Amara

Division of Internal Medicine,

Gastroenterology and Liver Diseases

Unit, EMMS, The Nazareth Hospital,

Nazareth 16100 , Israel

$\mathrm{Tel}+9724602885 \mathrm{I}$

Fax +972 4755905 I

Email amara.emms@gmail.com
Background: Nonalcoholic fatty liver disease (NAFLD) is an emerging condition and is constituted as a vital public health epidemic globally. This study evaluated the process of identification and documentation of NAFLD and metabolic syndrome in correlation with those diagnosed with obesity.

Methods: Participants included 352 patients older than 18 years who were diagnosed with fatty liver disease. We performed a cross-sectional study between August 2016 and September 2017. Categorical variables were extracted and analyzed using SPSS. The body mass index (BMI) was determined by the study staff and compared with the data retrieved from the family physician's database.

Results: Patients who presented documented BMI in their past medical history showed to be significantly higher than those without documentation of BMI $\left(29+4.4 \mathrm{vs} 25.7+4.6 \mathrm{~kg} / \mathrm{m}^{2}\right.$, $P<0.01)$. For instance, $54 \%$ of patients with NAFLD were documented in the electronic medical record (EMR) by the family physician, with higher documentation rate among males than females. Moreover, $72 \%$ qualified for documentation of metabolic syndrome, but only $5 \%$ were documented in their EMR. Patients with significant obesity and obesity-related conditions were more likely to have documentation in their EMR.

Discussion: Further analyses supported the conclusion that family physicians inadequately identify BMI in the EMR for overweight, obesity, metabolic syndrome, and fatty liver disease. Additional efforts are necessary to improve knowledge of proper identification of NAFLD and metabolic syndrome.

Keywords: metabolic syndrome, family physician, obesity, nonalcoholic fatty liver disease, nonalcoholic steatohepatitis

\section{Introduction}

Nonalcoholic fatty liver disease (NAFLD) is an emerging condition and is constituted as an important public health epidemic globally. ${ }^{1}$ The fatty liver disease may also represent a simple hepatic steatosis (HPs) that could gradually progress to nonalcoholic steatohepatitis (NASH), fibrosis, cirrhosis, and hepatocellular carcinoma. ${ }^{2,3}$ NAFLD is also one of the most common causes of incidental elevated liver enzymes in the developed world. The prevalence of NASH in Europe and the USA ranges from 14\% to $20 \%$; this increase in the prevalence is directly related to the obesity epidemic as seen in these populations. ${ }^{1}$ Diabetes mellitus, obesity, and hyperlipidemia are common components of the metabolic syndrome (MS), which is frequently associated with the NAFLD. Therefore, NAFLD is considered to be the hepatic manifestation of MS.,5 
In 2001, the National Cholesterol Education Program Adult Treatment Panel III (NCEP ATP III) devised a definition for MS, which was later updated by the American Heart Association and the National Heart Lung and Blood Institute in 2005. As advocated in the NCEP ATP III definition, MS is present if three or more of the following five criteria are met: waist circumference over 40 inches (men) or 35 inches (women), blood pressure over 130/85 mm Hg, fasting triglyceride (TG) level over $150 \mathrm{mg} / \mathrm{dL}$, fasting high-density lipoprotein (HDL) cholesterol level $<40 \mathrm{mg} / \mathrm{dL}$ (men) or $50 \mathrm{mg} / \mathrm{dL}$ (women), and fasting blood sugar over $100 \mathrm{mg} /$ dL. ${ }^{6}$ These components of MS appear to be risk factors for the development of HPs. ${ }^{7}$

The public health's rightful concern with the growing number of MS, obesity, and NASH/NAFLD has primary care providers continuing on the front lines of the obesity epidemic. Residency-based studies show an increase in clinical management actively taking action when obesity is included on the list of diagnosis in the electronic medical record (EMR) rather than when it is not included. Although EMRs can calculate body mass index (BMI) automatically, majority of physicians do not put this information on the problem list of their patients' EMR, similarly with the diagnosis of NASH/NAFLD and MS. ${ }^{8,9}$

Several studies have demonstrated that physicians often fail to identify, diagnose, and manage obesity, NAFLD/ $\mathrm{NASH}$, and MS in the EMR, although it is critical to their patients' health and assists other physicians in diagnosing future prognosis. This may stem from physicians' lack of knowledge and confidence to the treatment of obesity and NAFLD/NASH. Additionally, various impediments such as insufficient time and inadequate compensation for weight and lifestyle consultation may also cause failure of adequate identification. ${ }^{8,10}$ Studies have shown that this lack of identification for the treatment of obesity and NAFLD/NASH has had a profound medical and economic effect on patient and healthcare system. ${ }^{9}$ The aim of this study is to examine whether family physicians (FPs) document their patients $\mathrm{BMI}$ and diagnose obesity, MS, and NAFLD/NASH in their patients.

\section{Methods and materials}

Following the approval of the Helsinki ethical review board of EMMS (Edinburgh Medical Missionary Society) Nazareth Hospital, a cross-sectional study was conducted between August 2016 and September 2017. The study was performed at the liver unit of the EMMS Nazareth Hospital in Nazareth,
Israel. The data used are coded in order to keep the anonymity of the patients. The informed consent forms were waived due to the noninterventional study design.

The study included 352 consecutive NAFLD/NASH patients from the liver unit at EMMS Nazareth Hospital. Our inclusion criteria consisted of patients aged 18 years and older who were diagnosed with NAFLD/NASH. Exclusion criteria included patients $<18$ years of age and patients with other hepatic pathology or autoimmune phenotypes, such as alcoholic liver disease, drug-induced liver injury, autoimmune hepatitis, viral hepatitis, cholesteric liver disease, and metabolic/genetic liver disease. Patient exclusion was determined by our clinical laboratory, radiological and/or histological criteria/tests (serology of viral hepatitis A, B, and $\mathrm{C}$ ). In addition, autoimmune markers included ANA, anti-LKM, antismooth muscle protein electrophoresis, immune electrophoresis, and metabolic markers such as serum ceruloplasmin, 24-hour urine collection for copper, ferritin, iron, transferrin saturation, TSH, hemoglobin A1c (HbA1c), and $\alpha-1$ antitrypsin.

Extracted data included sociodemographic variables (such as age and gender), BMI (18.5-24.9 is normal weight, 25.0-29.9 overweight, 30.0-34.9 class I obesity, and 35.039.9 class II obesity), serum levels of alanine transaminase (ALT; normal range: 7-55 units/L), aspartate transaminase (AST; normal range: 10-40 units/L), low-density lipoprotein cholesterol (LDL; normal range: 100-129 mg/dL), HDL cholesterol (normal range: 40-59 mg/dL), TGs (normal range: $150-199 \mathrm{mg} / \mathrm{dL}$ ), insulin (normal range: $<25 \mathrm{mIU} / \mathrm{L}$ ) and insulin resistance index (HOMA-IR; normal range: $<2.60$ ), glycated hemoglobin (HbAlc; normal range: $<6 \%$ ), and C-reactive protein (CRP; normal range: $<3 \mathrm{mg} / \mathrm{dL}$ ). Extracted variables also included comorbidities (including diabetes mellitus, hypertension, dyslipidemia, and smoking) and medication for our database. The BMI was determined by the study staff (in $\mathrm{kg} / \mathrm{m}^{2}$ ) and compared with the data retrieved from the FPs database (electronic file or referral letter), including the BMI calculation and documentation as well as MS and NFLD/NASH documentation.

\section{Statistical analysis}

The extracted data were analyzed using SPSS version 19 (IBM Corporation, Armonk, NY, USA). The continuous variables are expressed as the mean \pm SD. The $\chi^{2}$ test was used to examine the differences in categorical variables. Analysis of variance or the Student's $t$-test was used for comparisons of continuous variables. Multivariate odds ratios (ORs) and 
95\% confidence interval (CIs) were obtained to determine the predictors on BMI calculations and documentation as well as NAFLD/NASH and MS documentation. $P<0.05$ was considered statistically significant.

\section{Results}

A total of 352 patients were included in the final analysis, and the mean age of patients was $49 \pm 13$ years. Of the 352 patients, 191 (54\%) were males and $161(46 \%)$ were females, with a mean BMI of $26.7 \pm 4.7 \mathrm{~kg} / \mathrm{m}^{2}$ for both groups. Our results examined 97 obese patients $(27 \%)$ with a BMI $>30 \mathrm{~kg} / \mathrm{m}^{2}$ and 144 overweight patients (41\%) with a BMI between 25 and $29.9 \mathrm{~kg} / \mathrm{m}^{2}$. The prevalence of comorbidities included hypertension at $37 \%$, hyperlipidemia at $42 \%$, diabetes mellitus at $21 \%$ (the percentage was similar and not clinically significant among obese and overweight subjects), and a total of $74 \%$ of patients used chronic medication.

Of the 352 participants, 97 were obese and 144 were recorded as overweight. FPs measured and documented the BMI of 43 of 97 (44\%) obese patients (54/97 [56\%] show no BMI documentation in the EMR) and 61 of 144 (42\%) overweight patients (83/144 [58\%] show no BMI documentation in the EMR). When we examined the EMR of the 43 obese patients, only eight of them were found to have obesity as a medical diagnosis in their problem list, with a documented BMI of $19 \%$. The mean BMI of patients with documented BMI was significantly higher than those without documentation $\left(29 \pm 4.4\right.$ vs $\left.25.7 \pm 4.6 \mathrm{~kg} / \mathrm{m}^{2}, P<0.0\right)$. Moreover, patients with BMI documentation were significantly older than those without documentation $(59 \pm 14.4$ vs $47.7 \pm 14.6, P<0.01)$. Results showed that BMI documentation was higher among men than women ( $43 \%$ among men vs $24 \%$ among women, $P<0.01)$, as well as in association with several comorbidities.

The diagnosis of NAFLD/NASH, documented in the EMR by the FPs in 193 of 352 patients (54\%), showed a higher documentation rate among males than females ( $37 \%$ vs $22 \%$, $P<0.05)$. The percentage of patients with NASH and NAFLD diagnosis documentation in the EMRs was not significantly affected by BMI. According to the staff classification, 254 of 352 patients $(72 \%)$ were qualified for documentation of MS, but only $13(5 \%)$ were documented in their EMR.

Extracted data results were ALT 80.61 \pm 31.33 units/L (78), AST 48.81-14.99 units/L (45), CRP 3.80-2.44 mg/dL (3.6), HDL 37.69-8.63 mg/dL (36), LDL 132.36-24.01 mg/ dL (139), TG 153.12-36.63 (152), insulin 24.71-5.21 IU/mL (26), HbA1c 5.38\%-0.63\% (5.4\%), and HOMA-IR 2.88-1.06 (2.8). To explore the independent effect of predictors of BMI, $\mathrm{MS}$, and NAFLD/NASH documentation, a stepwise forward likelihood ratio and a multivariate logistic regression were performed on all univariate predictors associated with BMI, MS, and NAFLD/NASH documentation. Older patients (OR: 2.35, 95\% CI: 1.28-5.31) and obese patients (OR: 2.05, 95\% CI: 1.78-5.49) were more likely to have BMI documentation as well as MS and NAFLD/NASH documentation. Patients with comorbid conditions such diabetes mellitus (OR: 4.48, 95\% CI: 2.34-8.52) and hypertension (OR: 3.75, 95\% CI: 1.98-8.67) were also more likely to have documentation of BMI, MS, and NAFLD/NASH in their EMR.

\section{Discussion}

NAFLD, the hepatic manifestation of MS, is a common liver disease recognized globally and has become an increasingly important health issue. ${ }^{1}$ The role of primary care physicians is critical in the diagnosis and treatment of MS, NAFLD, and obesity, to promote weight loss through lifestyle changes. ${ }^{2,3}$ In addition, large proportions of patients are regularly reviewed by primary care physicians and will also receive liver function test. ${ }^{5}$ NAFLD is regarded as a hepatic component of MS. This is due to the fact that most of the features in MS are commonly present in subjects with NAFLD - for example, $67 \%-71 \%$ of subjects being obese, $12 \%-37 \%$ having impaired fasting glucose, $57 \%-68 \%$ having dyslipidemia, and $36 \%-70 \%$ being hypertensive.

In addition, we are able to hypothesize that, within the expected high prevalence of undiagnosed NAFLD/NASH, there is an increase in the prevalence of liver cirrhosis. Our study shows that FPs calculated and documented BMI in minimal overweight and obese patients, as well as there is a lack of proper documentation of obesity, MS, and NAFLD/ NASH in patients' EMR and medical problem list.

Our findings are consistent with previous research that has been conducted, ${ }^{11,12}$ including the fact that missing BMI data generate challenges for treatments by FPs. Studies have shown that practicing FPs and resident physicians failed to identify and manage obesity in a large proportion of obese patients. ${ }^{11,12}$ According to our knowledge, this is the first study to examine the failures of FPs in documenting MS and NAFLD/NASH diagnosis, in accordance with the inadequate calculation and documentation of BMI.

There are several impediments to both BMI and MS documentation, as well as NAFLD/NASH documentation in EMR and patient problem list in family practices. The physician may have several limitations. First, lack of knowledge needed to diagnose and treat obesity, fatty liver, and other components of MS. Second, time restraints and lack of reimbursement within the healthcare system. ${ }^{8}$ Finally, 
limitations on behalf of the patient's part, including embarrassment, the social stigma associated with obesity, and lack of motivation. ${ }^{8,13}$

Several studies have found that weight counseling by physicians is effective and improves the patient's chance of losing weight and maintaining a physically active lifestyle. ${ }^{14,15}$ However, in our study population, BMI was documented in only $44 \%$ of obese and $42 \%$ of overweight patients. In addition, obesity as a medical diagnosis was found in only $19 \%$ of patients ( 8 of 43 ). Diagnosis of NAFLD/NASH was documented in only 193 of 352 patients $(54 \%)$ in their EMR, and only 13 of 254 patients (5\%) have documented the diagnosis of MS in their EMR. The documentation of obesity, MS, and NAFLD/NASH was more often in significantly obese patients, males and older patients with other comorbid conditions.

The predictors of BMI, NAFLD/NASH, and MS documentation are comorbidities such as diabetes mellitus and hypertension. This can be explained, in part, because management of these conditions includes maintenance of normal weight. A large study performed in a primary care setting also showed that FPs are more likely to manage obesity when patients have obesity-related disorders, such diabetes, hypertension, sleep apnea, and steatohepatitis.

Obesity with BMI > 30 was found to be another predictor of BMI, MS, and NAFLD/NASH documentation in our study. This observation was observed in other previous studies. ${ }^{12}$ One factor to consider is that FPs sometimes rely on their patient's external appearance when making the diagnosis of obesity or fatty liver and tend to manage the condition with patients who seem externally heavier. However, primary prevention is extremely important; by providing early intervention to those at risk for obesity, the process of weight gain could be stopped or even reversed before the development of obesity or MS/fatty liver-related complications or conditions appear. ${ }^{11}$

Our study consisted of several limitations. First, we have taken into account that selection bias was prevalent, as subjects were recruited from patients who visit the liver unit of EMMS hospital for follow-ups. Thus, they were more concerned about their health status. Second, the population size used was too small to accurately reflect some known risk factors to be associated with obesity and NASH/NAFLD, such as ethnicity and family history. Moreover, it is plausible that FPs may have provided weight counseling and lifestyle modification consult without determining patients BMI and documentation of the diagnosis of obesity, MS, or NAFLD/NASH in their EMR. That being said, our study design did not assess the waist circumference measurement by FPs, which is alternative to BMI determination. However, because the EMR has a designated field for height, weight, and automatically calculating BMI, it is unlikely that the FPs favored waist circumference measurement. Lastly, some oxidative stress markers were not measured in our study and may be considered in future prospective, large cohort studies.

In conclusion, FPs inadequately determined BMI as well as documentation of overweight, obesity, MS, and fatty liver disease in EMRs. Patients with significant obesity and obesityrelated complications were more likely to have such documentation. BMI measurements and diagnosis documentation of obesity, MS, and NAFLD/NASH are very important and should be incorporated into the FPs routine medical visits in order to facilitate primary and secondary prevention. While obesity, MS, and fatty liver disease are at epidemic levels worldwide, their identification and management by FPs should be optimized.

\section{Acknowledgments}

The abstract of this article was presented at the EASL International Liver Congress of 2017 as a poster presentation with interim findings. The poster's abstract was published in "Poster Abstracts" Journal of Hepatology: https://doi. org/10.1016/S0168-8278(17)31180-7.

\section{Disclosure}

The authors report no conflicts of interest in this work.

\section{References}

1. Bertolotti M, Lonardo A, Mussi C, et al. Nonalcoholic fatty liver disease and aging: epidemiology to management. World J Gastroenterol. 2014;20(39):14185-14204.

2. Ahmed M. Non-alcoholic fatty liver disease in 2015. World J Hepatol. 2015;7(11):1450-1459.

3. Rinella ME. Nonalcoholic fatty liver disease: a systematic review. JAMA. 2015;313(22):2263-2273.

4. Mendonça FM, de Sousa FR, Barbosa AL, et al. Metabolic syndrome and risk of cancer: which link? Metabolism. 2015;64(2):182-189.

5. Brunt EM, Janney CG, di Bisceglie AM, Neuschwander-Tetri BA, Bacon BR. Nonalcoholic steatohepatitis: a proposal for grading and staging the histological lesions. Am J Gastroenterol. 1999;94(9):2467-2474.

6. Huang PL. A comprehensive definition for metabolic syndrome. Dis Model Mech. 2009;2(5-6):231-237.

7. Esposito K, Capuano A, Giugliano D. Metabolic syndrome and cancer: holistic or reductionist? Endocrine. 2014;45(3):362-364.

8. Fogelman Y, Vinker S, Lachter J, Biderman A, Itzhak B, Kitai E. Managing obesity: a survey of attitudes and practices among Israeli primary care physicians. Int J Obes Relat Metab Disord. 2002;26(10): 1393-1397.

9. Thompson D, Wolf AM. The medical-care cost burden of obesity. Obes Rev. 2001;2(3):189-197.

10. Lyznicki JM, Young DC, Riggs JA. Council on Scientific Affairs, American Medical Association. Obesity: assessment and management in primary care. Am Fam Physician. 2001;63(11):2185-2196. 
11. Lemay CA, Cashman S, Savageau J, Fletcher K, Kinney R, LongMiddleton E. Underdiagnosis of obesity at a community health center. J Am Board Fam Pract. 2003;16(1):14-21.

12. Bardia A, Holtan SG, Slezak JM, Thompson WG. Diagnosis of obesity by primary care physicians and impact on obesity management. Mayo Clin Proc. 2007;82(8):927-932.

13. Kushner RF. Barriers to providing nutrition counseling by physicians: a survey of primary care practitioners. Prev Med. 1995;24(6):546-552.
14. Kant AK, Miner P. Physician advice about being overweight: association with self-reported weight loss, dietary, and physical activity behaviors of US adolescents in the National Health and Nutrition Examination Survey, 1999-2002. Pediatrics. 2007;119(1):e142-e147.

15. Kreuter MW, Chheda SG, Bull FC. How does physician advice influence patient behavior? Evidence for a priming effect. Arch Fam Med. 2000;9(5):426-433.
Diabetes, Metabolic Syndrome and Obesity: Targets and Therapy is an international, peer-reviewed open-access journal committed to the rapid publication of the latest laboratory and clinical findings in the fields of diabetes, metabolic syndrome and obesity research. Original research, review, case reports, hypothesis formation, expert
Dovepress

opinion and commentaries are all considered for publication. The manuscript management system is completely online and includes a very quick and fair peer-review system, which is all easy to use. Visit $\mathrm{http}: / / \mathrm{www}$. dovepress.com/testimonials.php to read real quotes from published authors.

Submit your manuscript here: https://www.dovepress.com/diabetes-metabolic-syndrome-and-obesity-targets-and-therapy-journal 